\title{
Power Control Algorithms in Wireless Communication
}

\author{
Sukhpreet Kaur Khangura \\ Student Mtech \\ Electronics \& Communication Engg \\ Baba Banda Singh Bahadur Engg. \\ College, Fatehgarh Sahib, Punjab.
}

\author{
Kiranpreet Kaur \\ Senior Lecturer \\ ECE, Depatrment \\ Baba Banda Singh Bahadur Engg. \\ College, Fatehgarh Sahib,Punjab.
}

\author{
R. S. Uppal \\ AP \& HOD \\ ECE, Depatrment \\ Baba Banda Singh Bahadur Engg. \\ College, Fatehgarh Sahib,Punjab
}

\begin{abstract}
Radio resources in wireless communication systems, implementing different multiple access techniques, must be wisely managed. This perspective is pivotal since the variations in propagation channel are very fast. This complexity in the cellular system periodically contributes to different interference levels, high or low, resulting in the degradation of the system capacity. Transmitter power control is an efficient technique to mitigate the effect of interference under fading conditions, combat the Near-Far problem and conserve the battery life. Thus, an effective implementation of different power control algorithms in cellular radio communication systems can offer a significant improvement in the Quality of Service (QoS) to all the users. Choice of an appropriate power control algorithm is of prime importance, as it should aim at increasing the overall efficiency of the system. In this paper different distributed power control algorithms, each suited for implementation under different cellular technologies, were studied extensively. Specifically, three distributed power control algorithms are compared through simulations on the basis of performance metrics like Carrier to Interference Ratio (CIR) and Outage for the downlink case.
\end{abstract}

\section{Categories and Subject Descriptors}

C.2 [Computer-Communication Networks]: Network Architecture and Design-Wireless communication

\section{General Terms}

Distance Based Power Control Algorithms, Distributed Balancing Power Control Algorithm,, Adaptive Step Sir based Power Control Algorithm.

\section{Keywords:}

CDMA, Centralized Power Control, Decentralized Power Control

\section{INTRODUCTION}

Wireless communications is one of the most active areas of technology development of our time. Wireless communications today covers a very wide array of applications .This development is being driven primarily by the transformation of what has been largely a medium for supporting voice telephony into a medium for supporting other services, such as the transmission of video, images, text, and data. The traditional resources that have been used to add capacity to wireless systems are radio bandwidth and transmitter power. Unfortunately, these two resources are among the most severely limited in the deployment of modern wireless networks: radio bandwidth because of the very tight situation with regard to useful radio spectrum and transmitter power because mobile and other portable services require the use of battery power, which is limited. These two resources are simply not growing or improving at rates that can support anticipated demands for wireless capacity

The largest and most noticeable part of the telecommunications business is telephony. The principal wireless component of telephony is mobile (i.e. cellular) telephony. The worldwide growth rate in cellular telephony is very aggressive and analysts report that the number of cellular telephony subscriptions worldwide has now surpassed the number of wire line (i.e. fixed) telephony subscriptions. These additional wireless technologies provide a basis for a very rich array of applications, including local telephony service, broadband Internet access and distribution of high-rate entertainment content such as highdefinition video and high-quality audio to the home, within the home, to automobiles and so on like $3 \mathrm{G}$, these technologies have spurred considerable research in signal processing for wireless.

These technologies are supported by a number of transmission and channel-assignment techniques, including time-division multiple access (TDMA), code-division multiple access (CDMA), and other spread-spectrum systems, orthogonal frequency-division multiplexing (OFDM) and other multi carrier systems and high-rate single-carrier systems [14]. These techniques are chosen primarily to address the physical properties of wireless channels, among the most prominent of which are multi path fading, dispersion and interference. To obtain maximal benefit from these transmission techniques, to exploit the diversity opportunities of the wireless channel and to mitigate the impairments of the wireless channel, advanced receiver signal processing techniques are of interest. These include channel equalization to combat dispersion, RAKE combining to exploit resolvable multi path, multi-user detection to mitigate multiple-access interference, suppression methods for co-channel interference, beam forming to exploit spatial diversity and space-time processing to jointly exploit temporal and spatial properties of the signaling environment.

Radio resource management (RRM) is the system level control of co-channel interference and other radio transmission 
characteristics in wireless communication systems, for example cellular networks, wireless networks and broadcasting systems. RRM involves strategies and algorithms for controlling parameters such as transmit power, channel allocation, handover criteria, modulation scheme, error coding scheme, etc. The objective is to utilize the limited radio spectrum resources and radio network infrastructure as efficiently as possible. Among different Radio Resource Management (RRM) techniques, power control, also known as TPC (Transmit Power Control), is one of the important 'interference suppression' techniques. The system capacity and performance are adversely affected and degraded by interference. Hence, power control plays a prominent role in an interference-limited system, which increases the efficiency by mitigating the adjacent and co-channel interference in the system. Power control in general is all about controlling the transmitted power both in downlink and uplink direction for different reasons. Main aim of the power control is to transmit the signal with lowest possible power level, which maintains the required quality of signal. Power of every transmitter is adjusted to the level required to meet the requested QoS. Determining the transmitter power level is a very sophisticated task due to dynamic variation of the radio channel. Whatever the radio environment is, the received power should be at an acceptable level.

There are several power control techniques and algorithms using the link quality measurements in both forward and reverse channels to adjust the power levels. There are also centralized and distributed power control algorithms. But the limited scope of this paper is to consider only some of the important distributed power control algorithms for evaluation.

\section{NEAR FAR PROBLEM}

The problem is best described by taking an example: Consider a receiver and two transmitters (one close to the receiver; the other far away). If both transmitters transmit simultaneously and at equal powers, then due to the inverse square law (Since, $\mathbf{P r}=\mathbf{P t}$ / $\left(\mathbf{4}^{*} \mathbf{P i} \mathbf{*} \mathbf{d} / \mathbf{l a m b d a}\right)^{\wedge} \mathbf{2}, \mathbf{P r}$ is received power, $\mathbf{P t}$ is the transmitted power, $\mathbf{d}$ is the distance between the stations, lambda is the wavelength of operation and $\mathbf{P i}$ is $3.1428 \ldots$.$) , the receiver will$ receive more power from the nearer transmitter. This makes the farther transmitter voice more difficult to understand. Since one transmission's signal is the other's noise the signal-to-noise ratio (SNR) for the farther transmitter is much lower. If the nearer transmitter transmits a signal that is orders of magnitude higher than the farther transmitter, then the SNR for the farther transmitter may be below detect ability and the farther transmitter may just as well not transmit. This effectively jams the communication channel. In CDMA systems, this is commonly solved by dynamic output power adjustment of the transmitters. That is, the closer transmitters use less power so that the SNR for all transmitters at the receiver is roughly the same. This sometimes can have a noticeable impact on battery life, which can be dramatically different depending on distance from the base station. As the propagation losses between BS and MS's are different according to individual communication distances, the received levels at the base station are different from each other when all mobile stations transmit their signals at the same power
Moreover, the received level fluctuates quickly due to fading. In order to maintain the strength of received signal level at BS, power control technique must be employed in CDMA systems .Unlike FDMA and TDMA, which are bandwidth limited multiple access, WCDMA is interference limited multiple access. In FDMA and TDMA, power control is applied to reduce inter-cell interference within the cellular system that arises from frequency reuse while in WCDMA systems the purpose of the power control is mainly to reduce the intra-cell interference. Due to the fact that in the WCDMA system the total bandwidth is shared simultaneously, other users can experienced as a noise like interference. In case power control mechanism is missing, common sharing of bandwidth creates a severe problem referred to as Near-Far effect

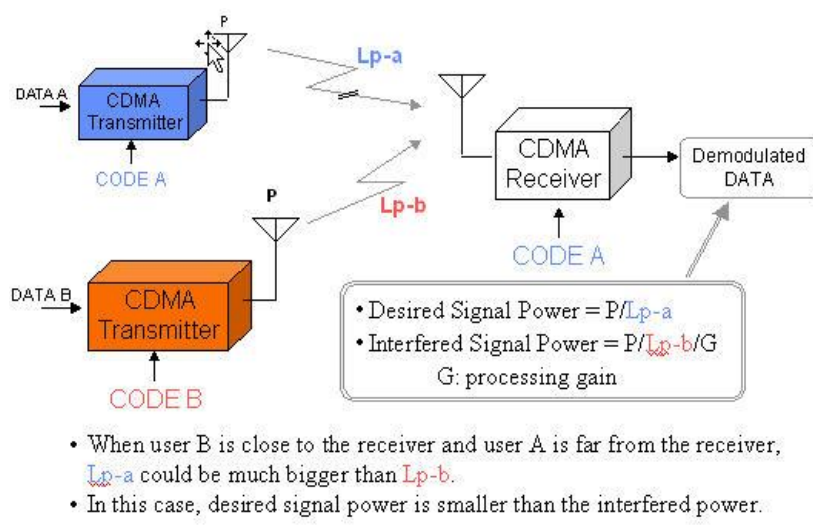

Figure 1. Near Far Problem in CDMA

\section{POWER CONTROL ALGORITHM}

Power control in 1st generation wireless networks was a relatively simple problem because of the enforced separation in resources i.e. FDMA networks used separate frequencies between different terminals with a significant gap/guard band between the two. This meant that the primary aim of power control was to ensure that there was sufficient received power so as to cross the noise threshold. Interference between terminals was a relatively small

problem. This still remains the case in satellite networks and other systems. However, when cellular networks came into operation the power control problem started taking center-stage. Since cellular networks aggressively re-use frequencies so as to maximize capacity, inter-cell interference started becoming the dominating source of impairment; as a result the network was "interference constrained" in capacity. Theoretically, power control can be formulated and solved as a convex optimization problem. In real-life power control is far more complex. This is due to relative movement of the remotes, lack of instantaneous information regarding individual link conditions and limited signaling capacity. We shall discuss these issues individually. In WCDMA, power control is employed in both the uplink and the downlink. The main target of the uplink power control is to mitigate the near-far problem by making the transmission power level received from all terminals as good as possible at the home cell for the same QoS. The mobile stations far away from the base station should transmit with considerably higher power than 
the mobiles close to the base station. Therefore uplink power control is for fine tuning of terminal transmission power, resulting in the mitigation of the intra-cell interference and nearfar effect. The situation is different in downlink direction. The downlink signals transmitted by one base station are orthogonal. Signals that are mutually orthogonal do not interfere with each other. However, it is impossible to achieve full orthogonal signals in typical usage environments. Signal reflections cause non orthogonal interference even if one base station is considered. Moreover signals sent from other base stations are, of course, non orthogonal and thus they increase the interference level taking into account that in a CDMA system the neighbor cells use the same downlink frequency carrier. This calls for downlink power control.

There are two basic types of power control:

1.Open Loop Power Control

2. Closed Loop Power Control

\subsection{Open Loop Power Control}

The open loop power control technique requires that the transmitting entity measures the channel interference and adjusts its transmission power accordingly. In this process, the UE estimates the transmission signal strength by measuring the received power level of the pilot signal from the BS in the downlink and adjusts its transmission power level in a way that is inversely proportional to the pilot signal power level. Consequently, the stronger the received pilot signal, the lower the UE transmitted power. This can be done quickly but the problem is that the interference estimation is done on the received signal and the transmitted signal probably uses the different frequency (in case of FDD), which differs from the received frequency by the system's duplex offset. As uplink and downlink fast fading (on different frequency carriers) do not correlate, this method gives the power values only on average.

\subsection{Closed Loop Power Control}

In the closed-loop power control technique, the quality measurements are done on the other end of the connection in the base station and the results are then sent back to mobile's transmitter so that it can adjust its transmitted power. This method gives much better results that the open loop method but it cannot react to quick changes in the channel conditions. The UTRA-FDD mode uses fast closed-loop power control technique both in uplink and downlink. In this method, the received SIR is measured over a 667 microseconds period (one TS), and based on that value, a decision is made about whether to increase or decrease the transmission power in the other end of the connection. The transmit power control (TPC) bits are sent in every time slot within uplink and downlink. All power control signals contain either an increase or decrease command. In uplink, When BS receives the UE signal it compares the signal strength with the pre-defined threshold value at the BS. If the UE transmission power exceeds the threshold value, the BS sends a Transmission Power Command to the UE to decrease its signal power. If received signal is lower than the threshold target the
BS sends a command to UE to increase its transmission power. In downlink, the roles of UE and BS are interchanged.

The fast-closed loop power control is also called the inner loop power control. Closed loop power control also includes another loop called outer loop.

The inner loop closed loop power control adjusts the transmitted power in order to keep the received SIR (SIRest) equal to given target (SIRtarget). If the used SIR value still gives a low quality bit stream, then SIRtarget must be increased. The SIR target is fixed according to the received BLER or BER. The setting of SIR target is done by the outer loop power control in order to match the required BLER. The required SIR depends on mobile speed and multipath profile. The inner loop and outer loop are visualized in figure below.

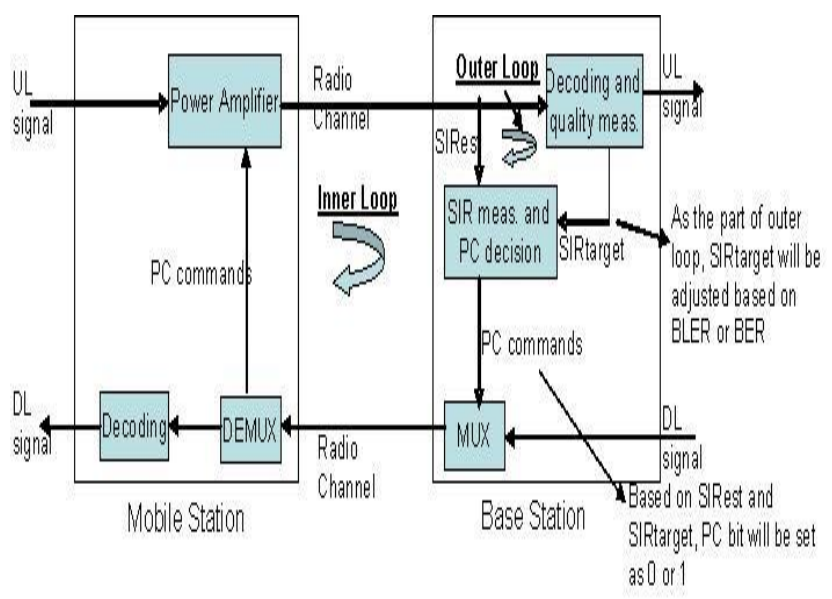

Figure2. Power control loops in CDMA

\subsubsection{Algorithms used in case of inner closed loop power control:}

The transmitted power is updated at each time slot according to which power control is done in wireless communication. It is increased or decreased by a fixed value as described below:

1. if SIRest > SIRtarget then TPC command to transmit is " 0 ", requesting a transmit power decrease.

2. if SIRest $<$ SIRtarget then TPC command to transmit is " 1 ", requesting a transmit power increase.

\subsection{Centralized vs. distributed power control}

Distributed power control algorithms are executed by the terminals and they work on the basis of the terminals own transmission power $\mathrm{P}_{\mathrm{i}}(\mathrm{n})$ in the nth time instant and the measured link condition $\gamma_{i}(n)$ corresponding to the same transmission. The algorithm works by updating the transmission power from the nth to the $(n+1)$ th instant by some arbitrary constant $c_{i}(n)$ i.e. $P_{i}(n+1)=c_{i}(n) P_{i}(n)$. It has been shown that for some conditions on the sequence $\mathrm{c}_{\mathrm{i}}$, the power vector converges asymptotically (in some cases weakly) to the optimal 
transmission vector. Different algorithms work on different mechanisms to compute the best value of $c_{i}(n)$

A key concern for distributed algorithms (to some little extent, centralized algorithms as well) is the convergence time; if the situation is stationary then convergence time does not matter; however, if the average network condition is changing, then the convergence time for the power control algorithm has to be faster than the rate of change. A second concern is the possibility of instability; specifically, sudden events causing a reaction from the entire active mobile population, leading to temporary network outages. How fast this kind of situation can be detected and mitigated is a property of the algorithm being used. Many networks which support distributed power control allow a central controller to damp the behavior of the terminals through broadcasts; thus trading off convergence time for stability. Some of the DPC algorithms considered are given below.

\section{PERFORMANCE ANALYSIS OF POWER CONTROL DOWNLINK ALGORITHMS}

We have carried out the performance analysis and comparison of the power control algorithms using Matlab 6.5.The comparison have been done by comparing Percentage of Mobiles in Outage versus No. of Mobiles in cells in downlink algorithms \& CIR versus number of iterations in Distributed Power control algorithms.

\subsection{DistanceBased Power Allocation Algorithm}

This is an open loop power control algorithm that uses the base station-to-mobile distance to allocate transmit powers for each mobile. The pseudo-code for the simulation is as follows. Fig. 3 plots the outage percentage against the number of mobiles.

Pseudo-code

1. Initialize number of iterations

2. Initialize number of mobiles

3. Initialize $\mathrm{d}_{\min }, \mathrm{R}, \mathrm{k}, \mathrm{n}$

4. for $\mathrm{i}=1$ to iterations

- Generate uniformly distributed vector of mobile-tobase station distance

- Initialize power

- for $\mathrm{j}=1$ to mobiles

- $\quad$ if $\mathrm{d}_{\mathrm{am}} \leq \mathrm{d}_{\mathrm{min}}$

- $\mathrm{p}_{\mathrm{m}}(\mathrm{j})=\mathrm{k}\left(\mathrm{d}_{\min } / \mathrm{R}\right)^{\mathrm{n}}$

- else

- $\quad p_{m}(j)=k\left(d_{a m}(j) / R\right)^{n}$

- $\quad$ end

- calculate $\mathrm{SIR}_{\text {observed(j) }}$ and compute outage

- $\quad$ end

5. Calculate the outage percentage using the outage counter and the number of mobiles.

6. Plot outage percentage versus mobiles

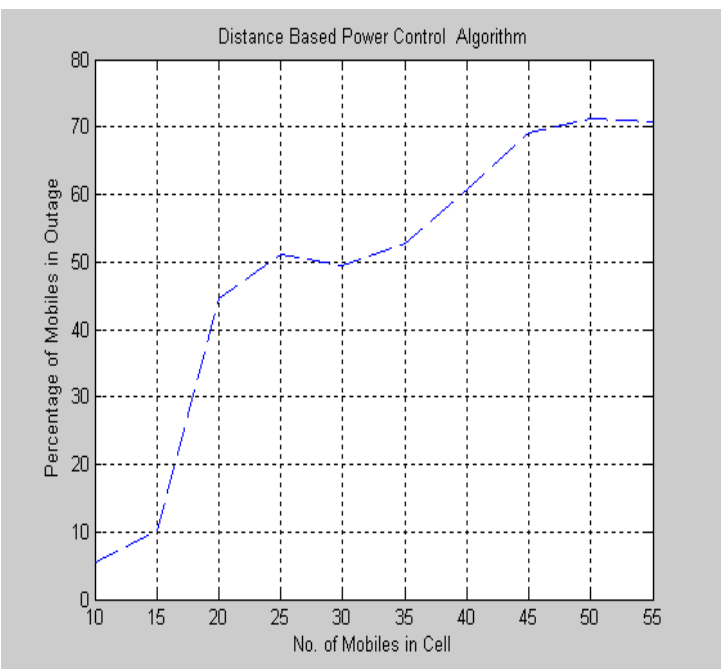

Figure 3 Outage Percentage of Mobiles in Outage versus No .of Mobiles in Cell

\subsection{Distributed Balancing Algorithm (DB)}

The distributed balancing algorithm calculates the optimal transmission power assignment for each mobile within the cell, taking into account all the neighboring cells. The scenario consists of a central cell surrounded by six other cells. This power is proportional to the ratio of the total received power of the mobile to the link gain between the base station and that mobile.

The pseudo-code for the simulation is as follows.

1. Initialize number of iterations

2. Initialize number of mobiles

3. for $\mathrm{i}=1$ to iterations

- Generate uniformly distributed vector of mobile-tobase station distance.

- Generate a vector of link gains of mobiles from their own base station gain.

- Generate a vector of link gains of mobiles from other base station gain.

- Initialize power

- Initialize DB correction coefficient $\mathrm{C}_{\mathrm{ik}}$

- for $\mathrm{j}=1$ to mobiles

- $\quad$ total received power $=\sum$ power $*$ gain $+6 * \sum$ power *gain'

- $\quad \mathrm{C}_{\mathrm{ik}}=$ total received power / gain

- $\quad \operatorname{power}(\mathrm{j})=\sum$ power $* \mathrm{C}_{\mathrm{ik}(\mathrm{j})} / \sum \mathrm{C}_{\mathrm{ik}}$

- end

- calculate SIRobserved(j) and compute outage

- end

end

4. Plot outage percentage versus mobiles 


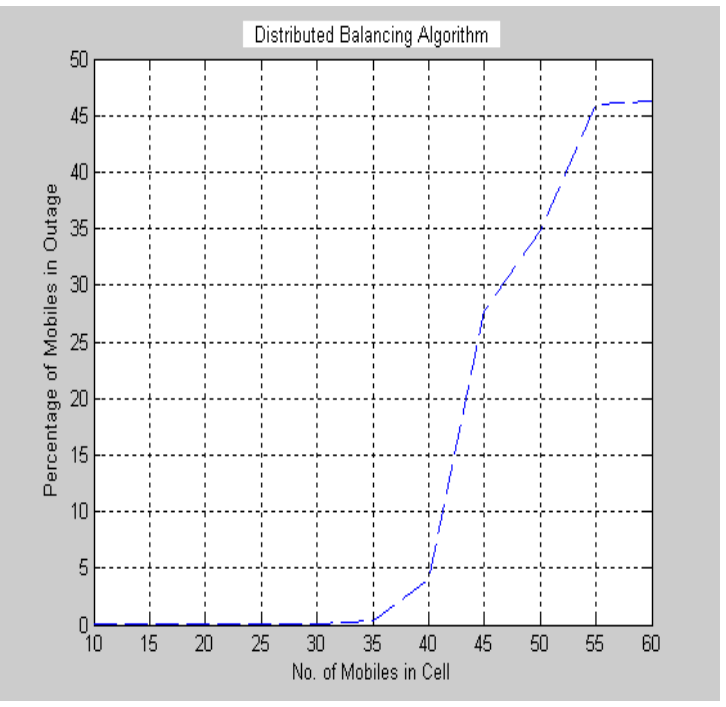

Figure 4. Outage Percentage of Mobiles in Outage versus No .of Mobiles in Cell

\subsection{Adaptive Step SIR based Power Control} ASPC is a variation of the MSPC algorithm that uses an adaptive step size to achieve faster convergence towards no outage. This algorithm uses the information from the previous iteration in order to adapt the step size accordingly.

The pseudo-code for the simulation is as follows.

\section{Pseudo-code}

1. Initialize number of iterations

2. Initialize number of mobiles

3. Generate uniformly distributed vector of mobile-to-base station distance

4. Generate a vector of link gains from each mobile to the base station based on a log-normal distribution with zero mean and standard deviation $=6 \mathrm{~dB}$

5. Allocate initial power for each mobile at the base station

6. Initialize $\operatorname{SIR}_{\text {threshold }}=-14 \mathrm{~dB}$

7. Initialize $\mu$ (increment factor), $v$ (decrement factor), $\delta$ (step size), flag (previous iteration feedback information)

8. for $\mathrm{i}=1$ to iterations

- for $\mathrm{j}=1$ to mobiles

- Calculate $\mathrm{SIR}_{\text {observed }}$ at each mobile

- Compare SIR $_{\text {observed }}$ with SIR threshold

- $\quad$ if $\left(\mathrm{SIR}_{\text {observed }} \leq \mathrm{SIR}_{\text {threshold }}\right)$

- Increment outage counter(i)

- $\quad$ if (previously in outage)

- $\quad$ Adjust (increase) power by $\mu \delta$

- else

- $\quad$ Adjust (increase) power by $\delta$

- $\quad$ Else if $\left(\mathrm{SIR}_{\text {observed }}>\mathrm{SIR}_{\text {threshold }}\right)$
- $\quad$ if (previously not in outage)

- $\quad$ Adjust (decrease) power by $v \delta$

- $\quad$ Else

- $\quad$ Adjust (decrease) power by $\delta$

- $\quad$ end

end

9. Calculate the outage percentage using outage counter and the number of mobiles

10. Plot outage percentage versus number of iteration

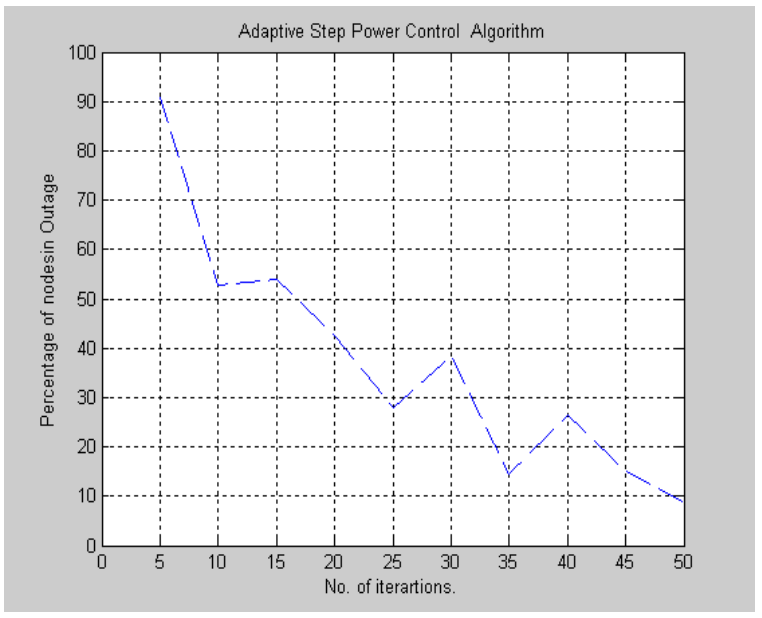

Figure 5. Outage Percentage of Mobiles in Outage versus No .of iterations

\section{CONCLUSION \& FUTURE WORK}

In this paper three existing downlink power control algorithms for CDMA systems were implemented and analyzed based on outage versus number of iterations. The Distributed Balancing (DB) power control algorithm was shown to give better results compared to Distance Based The performance of different algorithms can also be compared on the basis of metrics like BER and FER. Further, the system model developed can be extended to accommodate more number of users randomly moving in and out of the cell area. Even when the users per cell continuously change, the model should be easily adaptable to the changes. Power control along with base station assignment and beam forming, generally termed as 'combined power control' is also an interesting area of research. Areas like performance comparison of these combined power control algorithms, power control in MIMO and adaptive antenna systems and power control in WCDMA can be further explored in the light of this thesis and the results can easily be extended also to them.

In this paper we have studied three different existing downlink power control algorithms for CDMA systems were implemented and analyzed based on outage versus number of mobiles in cell and iterations. The Distributed Balancing (DB) power control algorithm was shown to give better results compared to Distance Based. The performance of different algorithms can also be compared on the basis of metrics like BER and FER. Further, the system model developed can be extended to accommodate more number of users randomly moving in and out of the cell area. 
Even when the users per cell continuously change, the model should be easily adaptable to the changes. Power control along with base station assignment and beam forming, generally termed as 'combined power control' is also an interesting area of research. Areas like performance comparison of these combined power control algorithms, power control in MIMO and adaptive antenna systems and power control in WCDMA can be further explored and results can easily be extended also to them.

\section{REFERNCES}

[1] GrandhiS.A.;Vijayan,R.;GoodmanD.J.;Zander,J.Vehicular Technology "Centralized Power Control in Cellular Radio Systems" IEEE Transactions on Volume 42, Issue 4, Nov 1993 Page(s):466 - 468

[2] Chung-Ju Chang and Fang-Ching Ren. " Downlink Power Control in DS/CDMA Cellular Mobile Radio Network" In Proceedings of 3rd International Conference on Universal Personal Communications,89-93, San Diego, CA, 1994.

[3] J. Viterbi. "CDMA: Principles of Spread Spectrum Communication". Addison-Wesley, Reading, MA, 1995.

[4] T.H. Lee and J.C. Lin, "A fully distributed power control algorithms for cellular mobile communication" IEEE Journal on selected areas in comm., vol.14, issue 4, pp.692697, May 1996

[5] D.kim, K.N.Chang and S.Kim "Efficient distributed power control for cellular mobile systems" IEEE Trans. on Veh. Technology, vol.46, pp.313319, May 1997

[6] Lei Song and Jack M. Holtzman“.CDMA Dynamic Downlink Power Control." In Proceedings of the Vehicular Technology Conference, volume 2 Ottawa, Canada, 1998.

[7] Tatcha Chulajata and Hyuck M. Kwon. "Combinations of Power Controls for CDMA 2000 Wireless Communications System." In Proceedings of the Vehicular Technology Conference (VTC'00), pages 638\{645,Boston, MA, 2000.
[8] Lout Nuaymi, Philippe Godlewski, and Xavier Lagrange. "A Power Control Algorithm for 3G WCDMA Systems." In Proceedings of European Wireless, Florence, Italy, 2002.

[9] Sarah Koskie and Zordan Gajic" Optimal SIR Based Power Strategies For Wireless CDMA networks" international journal Computing and Information Volume 4, Number 2, Pages 204- 218, 2008

[10] Asari, S.; Ho, P.; Jalan, A.; Velayudhan, "Performance Analysis Of The Forward Link In A Power Controlled CDMA Network" N.Vehicular Technology Conference, 1997 IEEE 47thVolume 3, Issue, 4-7 May 1997 Page(s):2118 - 2122 vol.3

[11] Sungmoon Shin, Hun Lee, Ki Chul Han, "The CDMA Mobile System Architecture” ,ETRI journal, volume 19, number3, Oct 1997

[12] W. M. Tam and F. C. M. Lau "Capacity Analysis of a CDMA Cellular System with Power Control Schemes" in $6^{\text {th }}$ IEEE International Conference on Universal Personal Communications (ICUPC'97), Pages 608-612, San Diego, CA, 1997

[13] S. C. Bang and Y. Han. "Performance of a Fast Forward Power Control Using Power Control Bits for the Reverse Power Control as Power Measurement" in the Vehicular Technology Conference (VTC'98), Pages 1414-1418, Ottawa, Canada, 1998.

[14] Man Young Rhee. "Cellular Mobile Communications CDMA Network and Security" Prentice-Hall, Upper Saddle River, NJ,1998

[15] Lei Song and Jack M. Holtzman "CDMA Dynamic Downlink Power Control" in Proceedings of the Vehicular Technology Conference (VTC'98), volume 2, Pages 11011105, Ottawa, Canada, 1998. 